\section{Mycotoxins and Phycotoxins}

\section{7-21 May 2004, Bethesda, Maryland, USA}

The 11th International Symposium on Mycotoxins and

Phycotoxins is the next symposium in a series initiated by the IUPAC Food Chemistry Commission. The first symposium was held in Kungaly, Sweden, in 1973; the nine symposia that followed were held in Pulawy, Poland; Paris, France; Lausanne, Switzerland; Vienna, Austria; Pretoria, South Africa; Tokyo, Japan; Mexico City, Mexico; Rome, Italy; and Guaraja, Brazil. The symposia have become the principal international, interdisciplinary meeting on mycotoxins and phycotoxins. The most recent, held in Brazil (May 2000), focused on the global significance of the toxins, occurrence, assay proficiency, toxicology and risk assessment.

The 11th symposium will assess progress or advances made since Brazil 2000 in relation to overall goals of the series and the evolving global perspective of food safety. The negative effect of mycotoxin and phycotoxin contamination of food commodities on health and global trade has been highlighted at various symposia. It is also understood that, without sound risk analysis, unsubstantiated health and safety claims become the basis of arbitrary regulations that could compromise food availability as well as international trade. The XI symposium will highlight the value of risk assessment and its regulatory use; laboratory quality assurance and quality control, and advances in sampling, accreditation and method validation procedures, particularly in developing countries. The XI symposium will also address novel molecular tools for detection, toxicogenomics and control strategies. There will be a "self assessment" of the IUPAC Symposium on Mycotoxins and Phycotoxins series from a historical and impact perspective.

\section{See Calendar on page 32 for contact information}

www to be announced

\section{Polymers and Organic Chemistry}

\section{8-23 July 2004, Prague, Czech Republic}

The program of 11th International Conference on Polymers and Organic Chemistry will focus on the following topics: polymer supported reagents, polymer catalysts, polymers in medicine and biochemistry, supports for fast chemistry, polymers for separations, electro- or light-sensitive functional polymers, polymers for environmental protection, processes within functional polymers, theoretical approaches to imprinted polymers, and novel functional polymers.
Summaries of contributions for consideration as oral presentations should be delivered no later than by 31 October 2003. Summaries of poster contributions are expected together with the final application forms before 31 March 2004. The venue for the conference will be a hotel-type facility operated by Charles University, located close to the airport, with excellent access to the city center (20 minutes by public transport).

See Calendar on page 33 for contact information

http://poc04.icpf.cas.cz

\section{Chemistry and Biological Chemistry of Vanadium}

\section{3-5 September 2004, Szeged, Hungary}

The 4th International Symposium on Chemistry and Biological Chemistry of Vanadium (V4) will follow the 7th European Conference on Bioinorganic Chemistry (EUROBIC7), in Garmisch Partenkirchen, Germany.

The scientific program covers the biological aspects of vanadium chemistry (biochemistry and therapeutic application of vanadium compounds), the field of inorganic chemistry, chemistry of catalysis, materials chemistry, and organic synthesis. The sym- posium will take place in three consecutive sections:

- biological aspects of vanadium chemistry

- inorganic chemistry of vanadium

- vanadium chemistry in catalysis and organic synthesis

The scientific program will include invited lectures and contributed papers (oral and poster). The official language of the symposium is English.

See Calendar on page 33 for contact information

www.staff-szeged.hu/ vanadium 